TRANSACTIONS OF THE

AMERICAN MATHEMATICAL SOCIETY

Volume 365, Number 8, August 2013, Pages 4475-4496

S 0002-9947(2013)05836-9

Article electronically published on January 4, 2013

\title{
THE RANGE OF LOCALIZATION OPERATORS AND LIFTING THEOREMS FOR MODULATION AND BARGMANN-FOCK SPACES
}

\author{
KARLHEINZ GRÖCHENIG AND JOACHIM TOFT
}

\begin{abstract}
We study the range of time-frequency localization operators acting on modulation spaces and prove a lifting theorem. As an application we also characterize the range of Gabor multipliers, and, in the realm of complex analysis, we characterize the range of certain Toeplitz operators on weighted Bargmann-Fock spaces. The main tools are the construction of canonical isomorphisms between modulation spaces of Hilbert-type and a refined version of the spectral invariance of pseudodifferential operators. On the technical level we prove a new class of inequalities for weighted gamma functions.
\end{abstract}

\section{INTRODUCTION}

The precise description of the range of a linear operator is usually difficult, if not impossible, because this amounts to a characterization of which operator equations are solvable. In this paper we study the range of an important class of pseudodifferential operators, so-called time-frequency localization operators, and we prove an isomorphism theorem between modulation spaces with respect to different weights.

The guiding example to develop an intuition for our results is the class of multiplication operators. Let $m \geq 0$ be a weight function on $\mathbb{R}^{d}$ and define the weighted space $L_{m}^{p}\left(\mathbb{R}^{d}\right)$ by the norm $\|f\|_{L_{m}^{p}}=\left(\int_{\mathbb{R}^{d}}|f(x)|^{p} m(x)^{p} d x\right)^{1 / p}=\|f m\|_{L^{p}}$. Let $\mathcal{M}_{a}$ be the multiplication operator defined by $\mathcal{M}_{a} f=a f$. Then $L_{m}^{p}$ is precisely the range of the multiplication operator $\mathcal{M}_{1 / m}$.

We will prove a similar result for time-frequency localization operators between weighted modulation spaces. To set up terminology, let $\pi(z) g(t)=e^{2 \pi i \xi \cdot t} g(t-x)$ denote the time-frequency shift by $z=(x, \xi) \in \mathbb{R}^{2 d}$ acting on a function $g$ on $\mathbb{R}^{d}$. The corresponding transform is the short-time Fourier transform of a function defined by

$$
V_{g} f(z)=\int_{\mathbb{R}^{d}} f(t) \overline{g(t-x)} e^{-2 \pi i \xi \cdot t} d t=\langle f, \pi(z) g\rangle .
$$

The standard function spaces of time-frequency analysis are the modulation spaces. The modulation space norms measure smoothness in the time-frequency space (phase space in the language of physics) by imposing a norm on the short-time

Received by the editors October 3, 2011 and, in revised form, March 22, 2012.

2010 Mathematics Subject Classification. Primary 46B03, 42B35, 47B35.

Key words and phrases. Localization operator, Toeplitz operator, Bargmann-Fock space, modulation space, Sjöstrand class, spectral invariance, Hermite function.

The first author was supported in part by the project P2276-N13 of the Austrian Science Fund (FWF). 
Fourier transform of a function $f$. As a special case we mention the modulation spaces $M_{m}^{p}\left(\mathbb{R}^{d}\right)$ for $1 \leq p \leq \infty$ and a non-negative weight function $m$. Let

$$
h(t)=2^{d / 4} e^{-\pi t^{2}}=2^{d / 4} e^{-\pi t \cdot t}, \quad t \in \mathbb{R}^{d}
$$

denote the (normalized) Gaussian. Then the modulation space $M_{m}^{p}\left(\mathbb{R}^{d}\right)$ is defined by the norm

$$
\|f\|_{M_{m}^{p}}=\left\|V_{h} f\right\|_{L_{m}^{p}}
$$

The localization operator $A_{m}^{g}$ with respect to the "window" $g$, usually some test function, and the symbol or multiplier $m$ is defined formally by the integral

$$
A_{m}^{g} f=\int_{\mathbb{R}^{2 d}} m(z) V_{g} f(z) \pi(z) g d z .
$$

Localization operators constitute an important class of pseudodifferential operators and occur under different names such as Toeplitz operators or anti-Wick operators. They were introduced by Berezin as a form of quantization [3], and are nowadays applied in mathematical signal processing for time-frequency masking of signals and for phase-space localization [12. An equivalent form occurs in complex analysis as Toeplitz operators on Bargmann-Fock space [8, 4, 5]. In hard analysis they are used to approximate pseudodifferential operators, in some proofs of the sharp Gårding inequality and the Fefferman-Phong inequality [24, 25, 26, 30, and in PDE [1]. For the analysis of localization operators with time-frequency methods we refer to [10] and the references given there; for a more analytic point of view we recommend [32, 33].

A special case of our main result can be formulated as follows. By an isomorphism between two Banach spaces $X$ and $Y$ we understand a bounded and invertible operator from $X$ onto $Y$.

Theorem 1.1. Let $m$ be a non-negative continuous symbol on $\mathbb{R}^{2 d}$ satisfying $m(w+z) \leq e^{a|w|^{b}} m(z)$ for $w, z \in \mathbb{R}^{2 d}, 0 \leq b<1$, and assume that $m$ is radial in each time-frequency coordinate. Then for suitable test functions $g$ the localization operator $A_{m}^{g}$ is an isomorphism from the modulation space $M_{\mu}^{p}\left(\mathbb{R}^{d}\right)$ onto $M_{\mu / m}^{p}\left(\mathbb{R}^{d}\right)$ for all $1 \leq p \leq \infty$ and moderate weights $\mu$.

We see that the range of a localization operator exhibits the same behavior as the multiplication operators. For the precise formulation with all assumptions stated we refer to Section 4. The above isomorphism theorem can also be intepreted as a lifting theorem, quite in analogy with the lifting property of Besov spaces 35. However, whereas Besov spaces $\dot{B}_{s}^{p, q}$ with different smoothness $s$ are isomorphic via Fourier multipliers, the lifting operators between modulation spaces are precisely the localization operators with the weight $m$. This case is more subtle because localization operators are not closed under composition, quite in contrast to Fourier multipliers.

The isomorphism theorem for localization operators stated above is preceded by many contributions, which were already listed in [21. In particular, in [11] it was shown that for moderate symbol functions with subexponential growth the localization operator is a Fredholm operator, i.e., it differs from an invertible operator only by a finite-rank operator. Here we show that such operators are isomorphisms between the corresponding modulation spaces, even under weaker conditions than 
needed for the Fredholm property. In the companion paper 21 we proved the isomorphism property for weight functions of polynomial type, i.e.,

$$
c(1+|z|)^{-N} \leq m(z) \leq C\left((1+|z|)^{N} .\right.
$$

The contribution of Theorem 1.1 is the extension of the class of possible weights. In particular, the isomorphism property also holds for subexponential weight functions of the form $m(z)=e^{a|z|^{b}}$ or $m(z)=e^{a|z| / \log (e+|z|)}$ for $a>0,0<b<1$, which are often considered in time-frequency analysis. We remark that this generality comes at the price of imposing the radial symmetry on the weight $m$. Therefore, our results are not applicable to all situations covered by 21, where no radial symmetry is required.

Although the extension to weights of ultra-rapid growth looks like a routine generalization, it is not. The proof of Theorem 1.1 for weights of polynomial type is based on a deep theorem of Bony and Chemin 7. They construct a one-parameter group of isomorphisms from $L^{2}\left(\mathbb{R}^{d}\right)$ onto the modulation spaces $M_{m^{t}}^{2}\left(\mathbb{R}^{d}\right)$ for $t \in \mathbb{R}$. Unfortunately, the pseudodifferential calculus developed in [7] requires polynomial growth conditions, and, to our knowledge, an extention to symbols of faster growth is not available.

On a technical level, our contribution is the construction of canonical isomorphisms between $L^{2}\left(\mathbb{R}^{d}\right)$ and the modulation spaces $M_{m}^{2}\left(\mathbb{R}^{d}\right)$ of Hilbert type. In fact, such an isomorphism is given by an anti-Wick operator, i.e., by a time-frequency localization operator with Gaussian window $h(t)=2^{d / 4} e^{-\pi t^{2}}$.

Theorem 1.2. Assume that $m$ is a continuous moderate weight function of at most exponential growth and radial in each time-frequency coordinate. Then the localization operator $A_{m}^{h}$ is an isomorphism from $L^{2}\left(\mathbb{R}^{d}\right)$ onto $M_{1 / m}^{2}\left(\mathbb{R}^{d}\right)$ and from $M_{m}^{2}\left(\mathbb{R}^{d}\right)$ onto $L^{2}\left(\mathbb{R}^{d}\right)$.

This theorem replaces the result of Bony and Chemin. Theorem 1.2 was proved by Shubin 27] for the special case $m(z)=\left(1+|z|^{2}\right)^{1 / 2}$. In fact, Shubin defined the "Shubin class" $M_{m}^{2}$ as the range of the localization operator $A_{1 / m}^{h}$. The Shubin class $A_{1 / m}^{h} L^{2}$ was identified with the modulation space in [6]. The novelty is that Theorem 1.2 also covers symbols of ultra-rapid growth. Its proof requires new arguments and most of our efforts. We take a time-frequency approach rather than using classical methods from pseudodifferential calculus. In the course of its proof we will establish new inequalities for weighted gamma functions of the form

$$
C^{-1} \leq \int_{0}^{\infty} \theta(\sqrt{x / \pi}) \frac{x^{n}}{n !} e^{-x} d x \int_{0}^{\infty} \frac{1}{\theta(\sqrt{x / \pi})} \frac{x^{n}}{n !} e^{-x} d x \leq C \text { for all } n \in \mathbb{N} \cup\{0\} .
$$

The method of proof for Theorem 1.1 may be of interest in itself. Once the canonical isomorphisms are in place (Theorem 1.2), the proof of Theorem 1.1 proceeds as follows. It is easy to establish that $A_{m}$ is an isomorphism from $M_{\sqrt{m}}^{2}$ to $M_{1 / \sqrt{m}}^{2}$, so the composition $A_{1 / m}^{g} A_{m}^{g}$ is an isomorphism on $M_{\sqrt{m}}^{2}$. Using the canonical isomorphisms of Theorem 1.2, one next shows that the operator $V=A_{\sqrt{m}}^{h} A_{1 / m}^{g} A_{m}^{g} A_{1 / \sqrt{m}}^{h}$ is an isomorphism on $L^{2}$ and that the (Weyl) symbol of this operator belongs to a generalized Sjöstrand class. After these technicalities we apply the machinery of spectral invariance of pseudodifferential operators from [18] to conclude that $V$ is invertible on all modulation spaces $M_{\mu}^{p}$ (with $\mu$ compatible with the conditions on 
$m$ and the window $g$ ). Since $V$ is a composition of three isomorphisms, we then deduce that $A_{m}^{g}$ is an isomorphism from $M_{\mu}^{p}$ onto $M_{\mu / m}^{p}$.

As an application we prove (i) a new isomorphism theorem for so-called Gabor multipliers, which are a discrete version of time-frequency localization operators, and (ii) an isomorphism theorem for Toeplitz operators between weighted Bargmann-Fock spaces of entire functions. To formulate this result more explicitly, for a non-negative weight function $\mu$ and $1 \leq p \leq \infty$, let $\mathcal{F}_{\mu}^{p}\left(\mathbb{C}^{d}\right)$ be the space of entire functions of $d$ complex variables defined by the norm

$$
\|F\|_{\mathcal{F}_{\mu}^{p}}^{p}:=\int_{\mathbb{C}^{d}}|F(z)|^{p} \mu(z)^{p} e^{-p \pi|z|^{2} / 2} d z<\infty,
$$

and let $P$ be the usual projection from $L_{\text {loc }}^{1}\left(\mathbb{C}^{d}\right)$ to entire functions on $\mathbb{C}^{d}$. The Toeplitz operator with symbol $m$ acting on a function $F$ is defined to be $T_{m} F=$ $P(m F)$. Then we show that the Toeplitz operator $T_{m}$ is an isomorphism from $\mathcal{F}_{\mu}^{p}\left(\mathbb{C}^{d}\right)$ onto $\mathcal{F}_{\mu / m}^{p}\left(\mathbb{C}^{d}\right)$ for every $1 \leq p \leq \infty$ and every moderate weight $\mu$.

The connection between Toeplitz operators on Bargmann-Fock space and localization operators is also used in $[13$.

The paper is organized as follows: In Section 2 we provide the precise definition of modulation spaces and localization operators, and we collect their basic properties. In particular, we investigate the Weyl symbol of the composition of two localization operators. In Section 3, which contains our main contribution, we construct the canonical isomorphisms and prove Theorem 1.2. In Section 4 we derive a refinement of the spectral invariance of pseudodifferential operators and prove the general isomorphism theorem, of which Theorem 1.1 is a special case. Finally in Section 5 we give applications to Gabor multipliers and Toeplitz operators.

\section{TIME-FREQUENCY ANALYSIS AND LOCALIZATION OPERATORS}

We first set up the vocabulary of time-frequency analysis. For the notation we follow the book [17]. For a point $z=(x, \xi) \in \mathbb{R}^{2 d}$ in phase space the time-frequency shift of a function $f$ is $\pi(z) f(t)=e^{2 \pi i \xi \cdot t} f(t-x), t \in \mathbb{R}^{d}$.

The short-time Fourier transform: Fix a non-zero function $g \in L_{l o c}^{1}\left(\mathbb{R}^{d}\right)$ which is usually taken in a suitable space of Schwartz functions. Then the shorttime Fourier transform of a function or distribution $f$ on $\mathbb{R}^{d}$ is defined to be

$$
V_{g} f(z)=\langle f, \pi(z) g\rangle \quad z \in \mathbb{R}^{2 d},
$$

provided the scalar product is well-defined for every $z \in \mathbb{R}^{2 d}$. Here $g$ is called a "window function". If $f \in L^{p}\left(\mathbb{R}^{d}\right)$ and $g \in L^{p^{\prime}}\left(\mathbb{R}^{d}\right)$ for the conjugate parameter $p^{\prime}=p /(p-1)$, then the short-time Fourier transform can be written in integral form as

$$
V_{g} f(z)=\int_{\mathbb{R}^{d}} f(t) \overline{g(t-x)} e^{-2 \pi i \xi \cdot t} d t .
$$

In general, the bracket $\langle\cdot, \cdot\rangle$ extends the inner product on $L^{2}\left(\mathbb{R}^{d}\right)$ to any dual pairing between a distribution space and its space of test functions, for instance $g \in \mathcal{S}\left(\mathbb{R}^{d}\right)$ and $f \in \mathcal{S}^{\prime}\left(\mathbb{R}^{d}\right)$, but time-frequency analysis often needs larger distribution spaces. 
Weight functions: We call a continuous, strictly positive weight function $m$ on $\mathbb{R}^{2 d}$ moderate if

$$
\sup _{z \in \mathbb{R}^{2 d}}\left(\frac{m(z+y)}{m(z)}, \frac{m(z-y)}{m(z)}\right):=v(y)<\infty \quad \text { for all } y \in \mathbb{R}^{2 d} .
$$

The resulting function $v$ is a submultiplicative weight function, i.e., $v$ is even and satisfies $v\left(z_{1}+z_{2}\right) \leq v\left(z_{1}\right) v\left(z_{2}\right)$ for all $z_{1}, z_{2} \in \mathbb{R}^{2 d}$, and then $m$ satisfies

$$
m\left(z_{1}+z_{2}\right) \leq v\left(z_{1}\right) m\left(z_{2}\right) \quad \text { for all } z_{1}, z_{2} \in \mathbb{R}^{2 d} .
$$

Given a submultiplicative weight function $v$ on $\mathbb{R}^{2 d}$, any weight satisfying condition (3) is called $v$-moderate. For a fixed submultiplicative function $v$ the set

$$
\mathcal{M}_{v}:=\left\{m \in L_{l o c}^{\infty}\left(\mathbb{R}^{2 d}\right): 0<m\left(z_{1}+z_{2}\right) \leq v\left(z_{1}\right) m\left(z_{2}\right) \forall z_{1}, z_{2} \in \mathbb{R}^{2 d}\right\}
$$

contains all $v$-moderate weights.

Several times we will use that every $v$-moderate weight $m \in \mathcal{M}_{v}$ satisfies the following bounds:

$$
\frac{1}{v\left(z_{1}-z_{2}\right)} \leq \frac{m\left(z_{1}\right)}{m\left(z_{2}\right)} \leq v\left(z_{1}-z_{2}\right) \quad \text { for all } z_{1}, z_{2} \in \mathbb{R}^{2 d} .
$$

This follows from (3) by replacing $z_{1}$ with $z_{1}-z_{2}$.

Modulation spaces $M_{m}^{p, q}$ for arbitrary weights: For the general definition of modulation spaces we choose the Gaussian function $h(t)=2^{d / 4} e^{-\pi t \cdot t}$ as the canonical window function. Then the short-time Fourier transform is defined for arbitrary elements in the Gelfand-Shilov space $\left(S_{1 / 2}^{1 / 2}\right)^{\prime}\left(\mathbb{R}^{d}\right)$ of generalized functions. The modulation space $M_{m}^{p, q}\left(\mathbb{R}^{d}\right), 1 \leq p, q<\infty$ consists of all elements $f \in\left(S_{1 / 2}^{1 / 2}\right)^{\prime}\left(\mathbb{R}^{d}\right)$ such that the norm

$$
\|f\|_{M_{m}^{p, q}}:=\left(\int_{\mathbb{R}^{d}}\left(\int_{\mathbb{R}^{d}}\left|V_{h} f(x, \xi)\right|^{p} m(x, \xi)^{p} d x\right)^{q / p} d \xi\right)^{1 / q}=\left\|V_{h} f\right\|_{L_{m}^{p, q}}
$$

is finite. If $p=\infty$ or $q=\infty$, we make the usual modification and replace the integral by the supremum norm $\|\cdot\|_{L^{\infty}}$. If $m=1$, then we usually write $M^{p, q}$ instead of $M_{m}^{p, q}$. We also set $M_{m}^{p}=M_{m}^{p, p}$ and $M^{p}=M^{p, p}$.

The reader who does not like general distribution spaces may interprete $M_{m}^{p, q}\left(\mathbb{R}^{d}\right)$ as the completion of the finite linear combinations of time-frequency shifts $\mathcal{H}_{0}=$ $\operatorname{span}\left\{\pi(z) h: z \in \mathbb{R}^{2 d}\right\}$ with respect to the $M_{m}^{p, q}$-norm for $1 \leq p, q<\infty$ and as a weak ${ }^{*}$-relative closure, when $p=\infty$ or $q=\infty$. These issues arise only for extremely rapidly decaying weight functions. If $m \geq 1$ and $1 \leq p, q \leq 2$, then $M_{m}^{p, q}\left(\mathbb{R}^{d}\right)$ is in fact a subspace of $L^{2}\left(\mathbb{R}^{d}\right)$. If $m$ is of polynomial type (cf. (1)), then $M_{m}^{p, q}\left(\mathbb{R}^{d}\right)$ is a subspace of tempered distributions. This is the case that is usually considered, although the theory of modulation spaces was developed from the beginning to include arbitrary moderate weight functions [14, 17, 19].

Norm equivalence: Definition (5) uses the Gauss function as the canonical window. The definition of modulation spaces, however, does not depend on the particular choice of the window. More precisely, if $g \in M_{v}^{1}, g \neq 0$, and $m \in \mathcal{M}_{v}$, then there exist constants $A, B>0$ such that

$$
A\|f\|_{M_{m}^{p, q}} \leq\left\|V_{g} f\right\|_{L_{m}^{p, q}} \leq B\|f\|_{M_{m}^{p, q}}=B\left\|V_{h} f\right\|_{L_{m}^{p, q}} .
$$


We will usually write

for the equivalent norms.

$$
\left\|V_{g} f\right\|_{L_{m}^{p, q}} \asymp\|f\|_{M_{m}^{p, q}}
$$

Localization operators: Given a non-zero window function $g \in M_{v}^{1}\left(\mathbb{R}^{d}\right)$ and a symbol or multiplier $m$ on $\mathbb{R}^{2 d}$, the localization operator $A_{m}^{g}$ is defined informally by

$$
A_{m}^{g} f=\int_{\mathbb{R}^{2 d}} m(z) V_{g} f(z) \pi(z) g d z,
$$

provided the integral exists. A useful alternative definition of $A_{m}^{g}$ is the weak definition

$$
\left\langle A_{m}^{g} f, k\right\rangle_{L^{2}\left(\mathbb{R}^{d}\right)}=\left\langle m V_{g} f, V_{g} k\right\rangle_{L^{2}\left(\mathbb{R}^{2 d}\right)} .
$$

While in general the symbol $m$ may be a distribution in a modulation space of the form $M_{1 / v}^{\infty}\left(\mathbb{R}^{2 d}\right)$ [10, 33], we will investigate only localization operators whose symbol is a moderate weight function.

Taking the short-time Fourier transform of (7), we find that

$$
V_{g}\left(A_{m}^{g} f\right)(w)=\int_{\mathbb{R}^{2 d}} m(z) V_{g} f(z)\langle\pi(z) g, \pi(w) g\rangle d z=\left(\left(m V_{g} f\right) \natural V_{g} g\right)(w),
$$

with the usual twisted convolution $\natural$ defined by

$$
(F \bigsqcup G)(w)=\int_{\mathbb{R}^{2 d}} F(z) G(w-z) e^{2 \pi i z_{1} \cdot\left(z_{2}-w_{2}\right)} d z .
$$

Since

$$
F \mapsto \int_{\mathbb{R}^{2 d}} F(z)\langle\pi(z) g, \pi(\cdot) g\rangle d z
$$

is the projection from arbitrary tempered distributions on $\mathbb{R}^{2 d}$ onto functions of the form $V_{g} f$ for some distribution $f$, the localization operator can be seen as the composition of a multiplication operator and the projection onto the space of shorttime Fourier transforms for the fixed window $g$. In this light, localization operators resemble the classical Toeplitz operators, which are multiplication operators followed by a projection onto analytic functions. Therefore they are sometimes called Toeplitz operators [21, 31, 33. If the window $g$ is chosen to be the Gaussian, then this formal similarity can be made more precise. See Proposition 5.5 .

2.1. Mapping properties of localization operators. The mapping properties of localization operators on modulation spaces closely resemble the mapping properties of multiplication operators between weighted $L^{p}$-spaces. The boundedness of localization operators has been investigated on many levels of generality [10, 32, 36]. We will use the following boundedness result from [11, 33.

Lemma 2.1. Let $m \in \mathcal{M}_{v}$ and $\mu \in \mathcal{M}_{w}$. Fix $g \in M_{v w}^{1}\left(\mathbb{R}^{d}\right)$. Then the localization operator $A_{1 / m}^{g}$ is bounded from $M_{\mu}^{p, q}\left(\mathbb{R}^{d}\right)$ to $M_{\mu m}^{p, q}\left(\mathbb{R}^{d}\right)$.

Remark. The condition on the window $g$ is required to make sense of $V_{g} f$ for $f$ in the domain space $M_{\mu}^{p, q}\left(\mathbb{R}^{d}\right)$ and of $V_{g} k$ for $k$ in the dual $M_{1 /(\mu m)}^{p^{\prime}, q^{\prime}}\left(\mathbb{R}^{d}\right)=\left(M_{\mu m}^{p, q}\right)^{\prime}$ of the target space $M_{\mu m}^{p, q}$ for the full range of parameters $p, q \in[1, \infty]$. For fixed $p, q \in[1, \infty]$ weaker conditions may suffice, because the norm equivalence (6) still holds after relaxing the condition $g \in M_{v}^{1}$ into $g \in M_{v}^{r}$ for $r \leq \min \left(p, p^{\prime}, q, q^{\prime}\right)$ [34. 
On a special pair of modulation spaces, $A_{1 / m}^{g}$ is even an isomorphism [21].

Lemma 2.2. Let $g \in M_{v}^{1}, m \in \mathcal{M}_{v}$, and set $\theta=m^{1 / 2}$. Then $A_{m}^{g}$ is an isomorphism from $M_{\theta}^{2}\left(\mathbb{R}^{d}\right)$ onto $M_{1 / \theta}^{2}\left(\mathbb{R}^{d}\right)$.

Likewise $A_{1 / m}$ is an isomorphism from $M_{1 / \theta}^{2}\left(\mathbb{R}^{d}\right)$ onto $M_{\theta}^{2}\left(\mathbb{R}^{d}\right)$. Consequently the composition $A_{1 / m}^{g} A_{m}^{g}$ is an isomorphism on $M_{\theta}^{2}\left(\mathbb{R}^{d}\right)$, and $A_{m}^{g} A_{1 / m}^{g}$ is an isomorphism on $M_{1 / \theta}^{2}\left(\mathbb{R}^{d}\right)$.

Lemma 2.2 is based on the equivalence

$$
\left\langle A_{m}^{g} f, f\right\rangle=\left\langle m,\left|V_{g} f\right|^{2}\right\rangle=\left\|V_{g} f \cdot \theta\right\|_{2}^{2} \asymp\|f\|_{M_{\theta}^{2}}^{2},
$$

and is proved in detail in [21, Lemma 3.4].

2.2. The symbol of $A_{1 / m}^{g} A_{m}^{g}$. The composition of localization operators is no longer a localization operator, but the product of two localization operators still has a well-behaved Weyl symbol. In the following we use the time-frequency calculus of pseudodifferential operators as developed in [18, 20. Compared to the standard pseudodifferential operator calculus it is more restrictive because it is related to the constant Euclidean geometry on phase space. On the other hand, it is more general because it works for arbitrary moderate weight functions (excluding exponential growth).

Given a symbol $\sigma(x, \xi)$ on $\mathbb{R}^{d} \times \mathbb{R}^{d} \simeq \mathbb{R}^{2 d}$, the corresponding pseudodifferential operator in the Weyl calculus $\operatorname{Op}(\sigma)$ is defined formally as

$$
\mathrm{Op}(\sigma) f(x)=\iint_{\mathbb{R}^{2 d}} \sigma\left(\frac{x+y}{2}, \xi\right) e^{2 \pi i(x-y) \cdot \xi} f(y) d y d \xi
$$

with a suitable interpretation of the integral. If $\mathcal{C}$ is a class of symbols, we write $\mathrm{Op}(\mathcal{C})=\{\mathrm{Op}(\sigma): \sigma \in \mathcal{C}\}$ for the class of all pseudodifferential operators with symbols in $\mathcal{C}$. For the control of the symbol of composite operators we will use the following characterization of the generalized Sjöstrand class from [18. For the formulation associate to a submultiplicative weight $v(x, \xi)$ on $\mathbb{R}^{2 d}$ the rotated weight on $\mathbb{R}^{4 d}$ defined by

$$
\tilde{v}(x, \xi, \eta, y)=v(-y, \eta) .
$$

For radial weights, to which we will restrict later, the distinction between $v$ and $\tilde{v}$ is unnecessary.

Theorem 2.3. Fix a non-zero $g \in M_{v}^{1}$. An operator $T$ possesses a Weyl symbol in $M_{\tilde{v}}^{\infty, 1}, T \in \mathrm{Op}\left(M_{\tilde{v}}^{\infty, 1}\right)$, if and only if there exists a (semi-continuous) function $H \in L_{v}^{1}\left(\mathbb{R}^{2 d}\right)$ such that

$$
|\langle T \pi(z) g, \pi(y) g\rangle| \leq H(y-z) \quad \text { for all } y, z \in \mathbb{R}^{2 d} .
$$

Remark. This theorem says the symbol class $M_{\tilde{v}}^{\infty, 1}$ is characterized by the offdiagonal decay of its kernel with respect to time-frequency shifts. This kernel is in fact dominated by a convolution kernel. The composition of operators can then be studied with the help of convolution relations. Clearly this is significantly easier than the standard approaches that work with the Weyl symbol directly and the twisted product between Weyl symbols. See 22] for results in this direction.

Theorem 2.4. Assume that $g \in M_{v^{s}}^{1}\left(\mathbb{R}^{d}\right), T \in \mathrm{Op}\left(M_{\tilde{v}^{s}}^{\infty, 1}\right)$ for $s \geq 1 / 2$, and $\theta \in \mathcal{M}_{v^{1 / 2}}$. Then $A_{\theta}^{g} T A_{1 / \theta}^{g} \in \mathrm{Op}\left(M_{\tilde{v}^{s-1 / 2}}^{\infty, 1}\right)$. 
Proof. We distinguish the window $g$ of the localization operator $A_{m}^{g}$ from the window $h$ used in the expression of the kernel $\langle T \pi(z) h, \pi(y) h\rangle$. Choose $h$ to be the Gaussian; then $h \in M_{v}^{1}$ for every submultiplicative weight $v$. Let us first write the kernel $\langle T \pi(z) h, \pi(y) h\rangle$ informally and later justify the convergence of the integrals. Recall that

$$
T\left(A_{1 / \theta}^{g} f\right)=T\left(\int_{\mathbb{R}^{2 d}} \theta(u)^{-1}\langle f, \pi(u) g\rangle \pi(u) g d u\right) .
$$

Then

$$
\begin{aligned}
& \left\langle A_{\theta}^{g} T A_{1 / \theta}^{g} \pi(z) h, \pi(y) h\right\rangle=\left\langle T A_{1 / \theta}^{g} \pi(z) h, A_{\theta}^{g} \pi(y) h\right\rangle \\
& =\iint_{\mathbb{R}^{4 d}} \frac{1}{\theta(u)}\langle\pi(z) h, \pi(u) g\rangle\left\langle T \pi(u) g, \pi\left(u^{\prime}\right) g\right\rangle \theta\left(u^{\prime}\right)\left\langle\pi(y) h, \pi\left(u^{\prime}\right) g\right\rangle d u d u^{\prime} .
\end{aligned}
$$

Now set

$$
G(z)=|\langle g, \pi(z) h\rangle|=\left|V_{h} g(z)\right| \text { and } G^{*}(z)=G(-z)
$$

and let $H$ be a dominating function in $L_{v}^{1}\left(\mathbb{R}^{2 d}\right)$ so that

$$
\left|\left\langle T \pi(u) g, \pi\left(u^{\prime}\right) g\right\rangle\right| \leq H\left(u^{\prime}-u\right) .
$$

Since time-frequency shifts commute up to a phase factor, we have

$$
|\langle\pi(z) h, \pi(u) g\rangle|=G(z-u) .
$$

Before substituting all estimates into (11), we recall that $\theta$ is $\sqrt{v}$-moderate by assumption, and so (4) says that

$$
\frac{\theta\left(u^{\prime}\right)}{\theta(u)} \leq v\left(u^{\prime}-u\right)^{1 / 2} \quad \text { for all } u, u^{\prime} \in \mathbb{R}^{2 d} .
$$

Now by (11) we get

$$
\begin{aligned}
\left|\left\langle A_{\theta}^{g} T A_{1 / \theta}^{g} \pi(z) h, \pi(y) h\right\rangle\right| & \\
& \leq \int_{\mathbb{R}^{2 d}} \int_{\mathbb{R}^{2 d}} \frac{\theta\left(u^{\prime}\right)}{\theta(u)} G(z-u) H\left(u^{\prime}-u\right) G\left(y-u^{\prime}\right) d u d u^{\prime} \\
& \leq \int_{\mathbb{R}^{2 d}} \int_{\mathbb{R}^{2 d}} G(z-u) v\left(u^{\prime}-u\right)^{1 / 2} H\left(u^{\prime}-u\right) G\left(y-u^{\prime}\right) d u d u^{\prime} \\
& =\left(G *\left(v^{1 / 2} H\right) * G^{*}\right)(z-y) .
\end{aligned}
$$

Thus the kernel of $A_{\theta}^{g} T A_{1 / \theta}^{g}$ is dominated by the function $G *\left(v^{1 / 2} H\right) * G^{*}$. By assumption $g \in M_{v^{s}}^{1}\left(\mathbb{R}^{d}\right)$ and thus $G \in L_{v^{s}}^{1}\left(\mathbb{R}^{2 d}\right)$, and $T \in \mathrm{Op}\left(M_{\tilde{v}^{s}}^{\infty, 1}\right)$ and thus $H \in L_{v^{s}}^{1}\left(\mathbb{R}^{2 d}\right)$. Then $v^{1 / 2} H \in L_{v^{s-1 / 2}}^{1}\left(\mathbb{R}^{2 d}\right)$. Consequently

$$
G *\left(v^{1 / 2} H\right) * G^{*} \in L_{v^{s}}^{1} * L_{v^{s-1 / 2}}^{1} * L_{v^{s}}^{1} \subseteq L_{v^{s-1 / 2}}^{1} .
$$

The characterization of Theorem 2.3 now implies that $A_{\theta}^{g} T A_{1 / \theta}^{g} \in \mathrm{Op}\left(M_{\tilde{v}^{s-1 / 2}}^{\infty, 1}\right)$.

Corollary 2.5. Assume that $g \in M_{v^{2} w}^{1}\left(\mathbb{R}^{d}\right)$ and $m \in \mathcal{M}_{v}$ and $w$ is an arbitrary submultiplicative weight. Then $A_{1 / m}^{g} A_{m}^{g} \in \operatorname{Op}\left(M_{\tilde{v} \tilde{w}}^{\infty, 1}\right)$. 
Proof. In this case $T$ is the identity operator and $\operatorname{Id} \in \mathrm{Op}\left(M_{v_{0}}^{\infty, 1}\right)$ for every submultiplicative weight $v_{0}(x, \xi, \eta, y)=v_{0}(-y, y)$. In particular, Id $\in \operatorname{Op}\left(M_{\widetilde{v}^{2}}^{\infty, 1}\right)$. Now replace the weight $\theta$ in Theorem 2.4 by $m$ and the condition $\theta \in \mathcal{M}_{v^{1 / 2}}$ by $m \in \mathcal{M}_{v}$ and modify the convolution inequality (12) in the proof of Theorem 2.4.

\section{Canonical isomorphisms Between modulation spaCes OF HILBERT-TYPE}

In 21 we have used a deep result of Bony and Chemin 7 regarding the existence of isomorphisms between modulation spaces of Hilbert-type and then extended those isomorphisms to arbitrary modulation spaces. Unfortunately the result of Bony and Chemin is restricted to weights of polynomial-type and does not cover weights moderated by superfast growing functions, such as $v(z)=e^{a|z|^{b}}$ for $0<$ $b<1$.

In this section we construct explicit isomorphisms between $L^{2}\left(\mathbb{R}^{d}\right)$ and the modulation spaces $M_{\theta}^{2}\left(\mathbb{R}^{d}\right)$ for a general class of weights. We will assume that the weights are radial in each time-frequency variable. Precisely, consider time-frequency variables

$$
(x, \xi) \simeq z=x+i \xi \in \mathbb{C}^{d} \simeq \mathbb{R}^{2 d}
$$

which we identify by

$$
\left(x_{1}, \xi_{1} ; x_{2}, \xi_{2} ; \ldots ; x_{d}, \xi_{d}\right)=\left(z_{1}, z_{2}, \ldots, z_{d}\right) \in \mathbb{C}^{d} \simeq \mathbb{R}^{2 d} .
$$

Then the weight function $m$ should satisfy

$$
m(z)=m_{0}\left(\left|z_{1}\right|, \ldots,\left|z_{d}\right|\right) \quad \text { for } z \in \mathbb{R}^{2 d}
$$

for some function $m_{0}$ on $\overline{\mathbb{R}}_{+}^{d}=[0, \infty)^{d}$. Without loss of generality, we may also assume that $m$ is continuous on $\mathbb{R}^{2 d}$. (Recall that only weights of polynomial-type occur in the lifting results in [21]. On the other hand, no radial symmetry is needed in 21].)

For each multi-index $\alpha=\left(\alpha_{1}, \ldots, \alpha_{d}\right) \in \mathbb{N}_{0}^{d}$ we denote the corresponding multivariate Hermite function by

$$
h_{\alpha}(t)=\prod_{j=1}^{d} h_{\alpha_{j}}\left(t_{j}\right), \quad \text { where } \quad h_{n}(x)=\frac{2^{1 / 4} \pi^{n / 2}}{n !^{1 / 2}} e^{\pi x^{2}} \frac{d^{n}}{d x^{n}}\left(e^{-2 \pi x^{2}}\right)
$$

is the $n$-th Hermite function in one variable with the normalization $\left\|h_{n}\right\|_{2}=1$.

Then the collection of all Hermite functions $h_{\alpha}, \alpha \geq 0$, is an orthonormal basis of $L^{2}\left(\mathbb{R}^{d}\right)$. By identifying $\mathbb{R}^{2 d}$ with $\mathbb{C}^{d}$ via (13), the short-time Fourier transform of $h_{\alpha}$ with respect to $h(t)=2^{d / 4} e^{-\pi t^{2}}$ is simply

$$
V_{h} h_{\alpha}(\bar{z})=e^{-\pi i x \cdot \xi}\left(\frac{\pi^{|\alpha|}}{\alpha !}\right)^{1 / 2} z^{\alpha} e^{-\pi|z|^{2} / 2}=e^{-\pi i x \cdot \xi} e_{\alpha}(z) e^{-\pi|z|^{2}} \quad \text { for } z \in \mathbb{C}^{d} .
$$

Remark. We mention that a formal Hermite expansion $f=\sum_{\alpha} c_{\alpha} h_{\alpha}$ defines a distribution in the Gelfand-Shilov space $\left(S_{1 / 2}^{1 / 2}\right)^{\prime}$ if and only if the coefficients satisfy $\left|c_{\alpha}\right|=\mathcal{O}\left(e^{\epsilon|\alpha|}\right)$ for every $\epsilon>0$. The Hermite expansion then converges in the weak* topology. Here we have used the fact that the duality $\left(S_{1 / 2}^{1 / 2}\right)^{\prime} \times S_{1 / 2}^{1 / 2}$ extends the 
$L^{2}$-form $\langle\cdot, \cdot\rangle$ on $S_{1 / 2}^{1 / 2}$, and likewise the duality of the modulation spaces $M_{\theta}^{2}\left(\mathbb{R}^{d}\right) \times$ $M_{1 / \theta}^{2}\left(\mathbb{R}^{d}\right)$. Consequently, the coefficient $c_{\alpha}$ of a Hermite expansion is uniquely determined by $c_{\alpha}=\left\langle f, h_{\alpha}\right\rangle$ for $f \in\left(S_{1 / 2}^{1 / 2}\right)^{\prime}$. See [23] for details.

In the following we take for granted the existence and convergence of Hermite expansions for functions and distributions in arbitrary modulation spaces. By $d \mu(z)=e^{-\pi|z|^{2}} d z$ we denote the Gaussian measure on $\mathbb{C}^{d}$.

Lemma 3.1. Assume that $\theta(z)=\mathcal{O}\left(e^{a|z|}\right)$ and that $\theta$ is radial in each coordinate.

(a) Then the monomials $z^{\alpha}, \alpha \geq 0$, are orthogonal in $L_{\theta}^{2}\left(\mathbb{C}^{d}, \mu\right)$.

(b) The finite linear combinations of the Hermite functions are dense in $M_{\theta}^{2}\left(\mathbb{R}^{d}\right)$.

By using polar coordinates $z_{j}=r_{j} e^{i \varphi_{j}}$, where $r_{j} \geq 0$ and $\varphi_{j} \in[0,2 \pi)$, we get

$$
z^{\alpha}=r^{\alpha} e^{i \alpha \cdot \varphi} \quad \text { and } \quad d z=r_{1} \cdots r_{d} d \varphi d r,
$$

and the condition on $\theta$ in Lemma 3.1 can be recast as

$$
\theta(z)=\theta_{0}(r)
$$

for some appropriate function $\theta_{0}$ on $[0, \infty)^{d}$, and $r=\left(r_{1}, \ldots, r_{d}\right)$ and $\varphi=\left(\varphi_{1}, \ldots, \varphi_{d}\right)$ as usual.

Proof. (a) This is well known and is proved in [12, 16. In order to be self-contained, we recall the arguments. By writing the integral over $\mathbb{R}^{2 d}$ in polar coordinates in each time-frequency pair, (16) and (17) give

$$
\int_{\mathbb{R}^{2 d}} z^{\alpha} \overline{z^{\beta}} \theta(z)^{2} e^{-\pi|z|^{2}} d z=\iint_{\mathbb{R}_{+}^{d} \times[0,2 \pi)^{d}} e^{i(\alpha-\beta) \cdot \varphi} r^{\alpha+\beta} e^{-\pi r^{2}} \theta_{0}(r)^{2} r_{1} \cdots r_{d} d \varphi d r .
$$

The integral over the angles $\varphi_{j}$ is zero, unless $\alpha=\beta$, whence the orthogonality of the monomials.

(b) Density: Assume on the contrary that the closed subspace in $M_{\theta}^{2}\left(\mathbb{R}^{d}\right)$ spanned by the Hermite functions is a proper subspace of $M_{\theta}^{2}\left(\mathbb{R}^{d}\right)$. Then there exists a nonzero $f \in\left(M_{\theta}^{2}\left(\mathbb{R}^{d}\right)\right)^{\prime}=M_{1 / \theta}^{2}\left(\mathbb{R}^{d}\right)$ such that $\left\langle f, h_{\alpha}\right\rangle=0$ for all Hermite functions $h_{\alpha} \in M_{\theta}^{2}\left(\mathbb{R}^{d}\right), \alpha \in \mathbb{N}_{0}^{d}$. Consequently the Hermite expansion of $f=\sum_{\alpha}\left\langle f, h_{\alpha}\right\rangle h_{\alpha}=$ 0 in $\left(S_{1 / 2}^{1 / 2}\right)^{\prime}$, which contradicts the assumption that $f \neq 0$.

Definition 1. The anti-Wick operator $J_{m}$ is the localization operator $A_{m}^{h}$ associated to the weight $m$ and to the Gaussian window $h=h_{0}$. Specifically,

$$
J_{m} f=\int_{\mathbb{R}^{2 d}} m(z)\langle f, \pi(z) h\rangle \pi(z) h d z .
$$

For $m=\theta^{2}$ we obtain

$$
\left\langle J_{m} f, f\right\rangle=\left\langle m V_{h} f, V_{h} f\right\rangle_{\mathbb{R}^{2 d}}=\left\|V_{h} f \theta\right\|_{2}^{2}=\|f\|_{M_{\theta}^{2}}^{2}
$$

whenever $f$ is in a suitable space of test functions.

Localization operators with respect to Gaussian windows have been used in several problems of analysis; see for instance [1, 24, 25, 27]. They have rather special properties. In view of the connection to the localization operators on the BargmannFock space (see below), this is to be expected. 
Theorem 3.2. If $\theta$ is a continuous, moderate function and radial in each timefrequency coordinate, then each of the mappings

$$
\begin{gathered}
J_{\theta}: M_{\theta}^{2}\left(\mathbb{R}^{d}\right) \rightarrow L^{2}\left(\mathbb{R}^{d}\right), \quad J_{\theta}: L^{2}\left(\mathbb{R}^{d}\right) \rightarrow M_{1 / \theta}^{2}\left(\mathbb{R}^{d}\right), \\
J_{1 / \theta}: M_{1 / \theta}^{2}\left(\mathbb{R}^{d}\right) \rightarrow L^{2}\left(\mathbb{R}^{d}\right), \quad J_{1 / \theta}: L^{2}\left(\mathbb{R}^{d}\right) \rightarrow M_{\theta}^{2}\left(\mathbb{R}^{d}\right)
\end{gathered}
$$

is an isomorphism.

The special case of the polynomial weight $\theta(z)=\left(1+|z|^{2}\right)^{1 / 2}$ was already proved in [27] with completely different methods. The extension to weights of non-polynomial growth is non-trivial and requires a number of preliminary results. In these investigations we will play with different coefficients of the form

$$
\tau_{\alpha}(\theta):=\left\langle J_{\theta} h_{\alpha}, h_{\alpha}\right\rangle,
$$

or, more generally,

$$
\tau_{\alpha, s}(\theta):=\tau_{\alpha}\left(\theta^{s}\right)=\left\langle J_{\theta^{s}} h_{\alpha}, h_{\alpha}\right\rangle=\int_{\mathbb{R}^{2 d}} \theta(z)^{s} \frac{\pi^{|\alpha|}}{\alpha !}\left|z^{\alpha}\right|^{2} e^{-\pi|z|^{2}} d z,
$$

when $\theta$ is a weight function and $s \in \mathbb{R}$. We note that the $\tau_{\alpha, s}(\theta)$ are strictly positive, since $\theta$ is positive. If $\theta \equiv 1$, then $\tau_{\alpha, s}(\theta)=1$, so we may consider the coefficients $\tau_{\alpha, s}(\theta) \alpha$ ! as weighted gamma functions.

Proposition 3.3 (Characterization of $M_{\theta}^{2}$ with Hermite functions). Let $\theta$ be $a$ moderate and radial function. Then

$$
\|f\|_{M_{\theta}^{2}}^{2}=\sum_{\alpha \geq 0}\left|\left\langle f, h_{\alpha}\right\rangle\right|^{2} \tau_{\alpha}\left(\theta^{2}\right) .
$$

Proof. Let $f=\sum_{\alpha>0} c_{\alpha} h_{\alpha}$ be a finite linear combination of Hermite functions. Since the short-time Fourier transform of $f$ with respect to the Gaussian $h$ is given by

$$
V_{h} f(\bar{z})=\sum c_{\alpha} V_{h} h_{\alpha}(\bar{z})=e^{-\pi i x \cdot \xi} \sum_{\alpha \geq 0} c_{\alpha} e_{\alpha}(z) e^{-\pi|z|^{2} / 2}
$$

in view of (15), definition (18) gives

$$
\begin{aligned}
\|f\|_{M_{\theta}^{2}}^{2} & =\int_{\mathbb{R}^{2 d}}\left|V_{h} f(z)\right|^{2} \theta(z)^{2} d z \\
& =\sum_{\alpha, \beta \geq 0} c_{\alpha} \overline{c_{\beta}} \int_{\mathbb{R}^{2 d}} e_{\alpha}(z) \overline{e_{\beta}(z)} \theta(z)^{2} e^{-\pi|z|^{2}} d z=\sum_{\alpha \geq 0}\left|c_{\alpha}\right|^{2} \tau_{\alpha}\left(\theta^{2}\right) .
\end{aligned}
$$

In the latter equalities it is essential that the weight $\theta$ is radial in each timefrequency coordinate so that the monomials $e_{\alpha}$ are orthogonal in $L_{\theta}^{2}\left(\mathbb{C}^{d}, \mu\right)$.

In the next proposition, which is due to Daubechies [12, we represent the antiWick operator by a Hermite expansion.

Proposition 3.4. Let $\theta$ be a moderate, continuous weight function on $\mathbb{R}^{2 d}$ that is radial in each time-frequency coordinate.

Then the Hermite function $h_{\alpha}$ is an eigenfunction of the localization operator $J_{\theta}$ with eigenvalue $\tau_{\alpha}(\theta)$ for $\alpha \in \mathbb{N}_{0}^{d}$, and $J_{\theta}$ possesses the eigenfunction expansion

$$
J_{\theta} f=\sum_{\alpha \geq 0} \tau_{\alpha}(\theta)\left\langle f, h_{\alpha}\right\rangle h_{\alpha} \quad \text { for all } f \in L^{2}\left(\mathbb{R}^{d}\right) .
$$


Proof. By Lemma 3.1(a) we find that, for $\alpha \neq \beta$,

$$
\begin{aligned}
\left\langle J_{\theta} h_{\beta}, h_{\alpha}\right\rangle & =\int_{\mathbb{C}^{d}} \theta(z) V_{h} h_{\beta}(z) \overline{V_{h} h_{\alpha}(z)} d z \\
& =\int_{\mathbb{C}^{d}} \theta(z) e_{\beta}(z) \overline{e_{\alpha}(z)} e^{-\pi|z|^{2}} d z=0 .
\end{aligned}
$$

This implies that $J_{\theta} h_{\alpha}=c h_{\alpha}$, and therefore $c=c\left\langle h_{\alpha}, h_{\alpha}\right\rangle=\left\langle J_{\theta} h_{\alpha}, h_{\alpha}\right\rangle=\tau_{\alpha}(\theta)$.

For a (finite) linear combination $f=\sum_{\beta \geq 0} c_{\beta} h_{\beta}$, we obtain

$$
\begin{aligned}
J_{\theta} f=\sum_{\alpha \geq 0}\left\langle J_{\theta} f, h_{\alpha}\right\rangle h_{\alpha}=\sum_{\alpha \geq 0} \sum_{\beta \geq 0} c_{\beta}\left\langle J_{\theta} h_{\beta}, h_{\alpha}\right\rangle h_{\alpha} \\
=\sum_{\alpha \geq 0} \sum_{\beta \geq 0} \tau_{\beta}(\theta) \delta_{\alpha, \beta} c_{\beta} h_{\alpha}=\sum_{\alpha \geq 0} \tau_{\alpha}(\theta) c_{\alpha} h_{\alpha} .
\end{aligned}
$$

The proposition follows because the Hermite functions span $M_{1 / \theta}^{2}\left(\mathbb{R}^{d}\right)$ and because the coefficients of a Hermite expansion are unique and given by $c_{\alpha}=\left\langle f, h_{\alpha}\right)$.

Corollary 3.5. If $\theta$ is moderate and radial in each coordinate, then $J_{\theta}: L^{2}\left(\mathbb{R}^{d}\right) \rightarrow$ $M_{1 / \theta}^{2}\left(\mathbb{R}^{d}\right)$ is one-to-one and possesses dense range in $M_{1 / \theta}^{2}\left(\mathbb{R}^{d}\right)$.

Proof. The coefficients in $J_{\theta} f=\sum_{\alpha \geq 0} \tau_{\alpha}(\theta)\left\langle f, h_{\alpha}\right\rangle h_{\alpha}$ are unique. If $J_{\theta} f=0$, then $\tau_{\alpha}(\theta)\left\langle f, h_{\alpha}\right\rangle=0$, and since $\tau_{\alpha}(\theta)>0$ we obtain $\left\langle f, h_{\alpha}\right\rangle=0$ and thus $f=0$. Clearly the range of $J_{\theta}$ in $M_{1 / \theta}^{2}\left(\mathbb{R}^{d}\right)$ contains the finite linear combinations of Hermite functions, and these are dense in $M_{1 / \theta}^{2}\left(\mathbb{R}^{d}\right)$ by Lemma 3.1(b).

To show that $J_{\theta}$ maps $L^{2}\left(\mathbb{R}^{d}\right)$ onto $M_{1 / \theta}^{2}\left(\mathbb{R}^{d}\right)$ is much more subtle. For this we need a new type of inequality valid for the weighted gamma functions in (20). By Proposition 3.4 the number $\tau_{\alpha, s}(\theta)$ is exactly the eigenvalue of the localization operator $J_{\theta^{s}}$ corresponding to the eigenfunction $h_{\alpha}$.

Proposition 3.6. If $\theta \in \mathcal{M}_{w}$ is continuous and radial in each time-frequency coordinate, then the mapping $s \mapsto \tau_{\alpha, s}(\theta)$ is "almost multiplicative". This means that for every $s, t \in \mathbb{R}$ there exists a constant $C=C(s, t)$ such that

$$
C^{-1} \leq \tau_{\alpha, s}(\theta) \tau_{\alpha, t}(\theta) \tau_{\alpha,-s-t}(\theta) \leq C \quad \text { for all multi-indices } \alpha .
$$

Proof. The upper bound is easy. By Proposition 3.4 the Hermite function $h_{\alpha}$ is a common eigenfunction of $J_{\theta^{s}}, J_{\theta^{t}}$, and $J_{\theta^{-s-t}}$. Since the operator $J_{\theta^{s}} J_{\theta^{t}} J_{\theta^{-s-t}}$ is bounded on $L^{2}\left(\mathbb{R}^{d}\right)$ by repeated application of Lemma 2.1, we obtain that

$$
\tau_{\alpha, s}(\theta) \tau_{\alpha, t}(\theta) \tau_{\alpha,-s-t}(\theta)=\left\|J_{\theta^{s}} J_{\theta^{t}} J_{\theta^{-s-t}} h_{\alpha}\right\|_{L^{2}} \leq C\left\|h_{\alpha}\right\|_{L^{2}}=C
$$

for all $\alpha \geq 0$. The constant $C$ is the operator norm of $J_{\theta^{s}} J_{\theta^{t}} J_{\theta^{-s-t}}$ on $L^{2}\left(\mathbb{R}^{d}\right)$.

For the lower bound we rewrite the definition of $\tau_{\alpha, s}(\theta)$ and make it more explicit by using polar coordinates $z_{j}=r_{j} e^{i \varphi_{j}}, r_{j} \geq 0, \varphi_{j} \in[0,2 \pi)$, in each variable. Then by assumption $\theta(z)=\theta_{0}(r)$ for some continuous moderate function $\theta_{0}$ on $\mathbb{R}_{+}^{d}$, and 
we obtain

$$
\begin{gathered}
\tau_{\alpha, s}(\theta)=\int_{\mathbb{R}^{2 d}} \theta(z)^{s} \frac{\pi^{|\alpha|}}{\alpha !}\left|z^{\alpha}\right|^{2} e^{-\pi|z|^{2}} d z \\
=(2 \pi)^{d} \int_{\mathbb{R}_{+}^{d}} \theta_{0}(r) \frac{\pi^{|\alpha|}}{\alpha !} r^{2 \alpha} e^{-\pi|r|^{2}} r_{1} \cdots r_{d} d r \\
=(2 \pi)^{d} \int_{0}^{\infty} \ldots \int_{0}^{\infty} \theta_{0}\left(r_{1}, \ldots, r_{d}\right) \prod_{j=1}^{d} \frac{1}{\alpha_{j} !}\left(\pi r_{j}^{2}\right)^{\alpha_{j}} e^{-\pi r_{j}^{2}} r_{1} \cdots r_{d} d r_{1} \cdots d r_{d} \\
=\int_{0}^{\infty} \ldots \int_{0}^{\infty} \theta_{0}\left(\sqrt{u_{1} / \pi}, \ldots, \sqrt{u_{d} / \pi}\right) \prod_{j=1}^{d} \frac{u_{j}^{\alpha_{j}}}{\alpha_{j} !} e^{-u_{j}} d u_{1} \cdots d u_{d} .
\end{gathered}
$$

We first focus on a single factor in the integral. The function $f_{n}(x)=x^{n} e^{-x} / n$ ! takes its maximum at $x=n$, and

$$
f_{n}(n)=\frac{1}{n !} n^{n} e^{-n}=(2 \pi n)^{-1 / 2}\left(1+\mathcal{O}\left(n^{-1}\right)\right)
$$

by Stirling's formula. Furthermore, $f_{n}$ is almost constant on the interval $[n-$ $\sqrt{n} / 2, n+\sqrt{n} / 2$ ] of length $\sqrt{n}$. On this interval the minimum of $f_{n}$ is taken at one of the endpoints $n \pm \sqrt{n} / 2$, where the value is

$$
f_{n}(n \pm \sqrt{n} / 2)=\frac{1}{n !}(n \pm \sqrt{n} / 2)^{n} e^{-(n \pm \sqrt{n} / 2)}=\frac{1}{n !} \frac{n^{n}}{e^{n}}\left(1 \pm \frac{1}{2 \sqrt{n}}\right)^{n} e^{\mp \sqrt{n} / 2} .
$$

Since

$$
\lim _{n \rightarrow \infty} \frac{(2 \pi n)^{1 / 2}\left(\frac{n}{e}\right)^{n}}{n !}=1 \text { and } \lim _{n \rightarrow \infty}\left(1 \pm \frac{1}{2 \sqrt{n}}\right)^{n} e^{\mp \sqrt{n} / 2}=e^{-1 / 8}
$$

by Stirling's formula and straightforward applications of Taylor's formula, we find that

$$
f_{n}(x) \geq \frac{c}{\sqrt{n}} \quad \text { for } x \in[n-\sqrt{n} / 2, n+\sqrt{n} / 2] \text { and all } n \geq 1 .
$$

For $n=0$ we use the inequality $f_{0}(x) \geq e^{-1 / 2}$ for $x \in[0,1 / 2]$.

Now consider the products of the $f_{n}$ 's occuring in the integral above. For $\alpha=$ $\left(\alpha_{1}, \ldots, \alpha_{d}\right) \in \mathbb{N}_{0}^{d}$ define the boxes

$$
C_{\alpha}=\prod_{j=1}^{d}\left[\alpha_{j}-2^{-1} \sqrt{\alpha_{j}}, \alpha_{j}+2^{-1} \max \left(\sqrt{\alpha_{j}}, 1\right)\right] \subseteq \mathbb{R}^{d},
$$

with volume $\operatorname{vol}\left(C_{\alpha}\right)=\prod_{j=1}^{d} \sqrt{\max \left(2^{-1}, \alpha_{j}\right)}$. Consequently, on the box $C_{\alpha}$ we have

$$
\prod_{j=1}^{d} \frac{u_{j}^{\alpha_{j}}}{\alpha_{j} !} e^{-u_{j}} \geq C_{0} \prod_{j=1}^{d} \frac{1}{\sqrt{\max \left(2^{-1}, \alpha_{j}\right)}}=C_{0}\left(\operatorname{vol} C_{\alpha}\right)^{-1}
$$

for some constant $C_{0}>0$ which is independent of $\alpha \in \mathbb{N}_{0}^{d}$. 
Next, to take into account the coordinate change in (25), we define the box

$$
D_{\alpha}=\prod_{j=1}^{d}\left[\frac{\left(\alpha_{j}-2^{-1} \sqrt{\alpha_{j}}\right)^{1 / 2}}{\sqrt{\pi}}, \frac{\left(\alpha_{j}+2^{-1} \max \left(\sqrt{\alpha_{j}}, 1\right)\right)^{1 / 2}}{\sqrt{\pi}}\right] \subseteq \mathbb{R}^{d} .
$$

Furthermore, the length of each edge of $D_{\alpha}$ is

$$
\pi^{-1 / 2}\left(\left(\alpha_{j}+\sqrt{\alpha_{j}} / 2\right)^{1 / 2}-\left(\alpha_{j}-\sqrt{\alpha_{j}} / 2\right)^{1 / 2}\right) \leq \pi^{-1 / 2}
$$

when $\alpha_{j} \geq 1$, and likewise for $\alpha_{j}=0$. Consequently,

$$
\text { if } z_{1}, z_{2} \in D_{\alpha} \text {, then } z_{1}-z_{2} \subseteq\left[-\pi^{-1 / 2}, \pi^{-1 / 2}\right]^{d} .
$$

After these preparations we start the lower estimate of $\tau_{\alpha, s}(\theta)$. Using (27) we obtain

$$
\begin{aligned}
\tau_{\alpha, s}(\theta) & =\int_{0}^{\infty} \cdots \int_{0}^{\infty} \theta_{0}\left(\sqrt{u_{1} / \pi}, \ldots, \sqrt{u_{d} / \pi}\right)^{s} \prod_{j=1}^{d} \frac{u_{j}^{\alpha_{j}}}{\alpha_{j} !} e^{-u_{j}} d u_{1} \cdots d u_{d} \\
& \geq C_{0} \frac{1}{\operatorname{vol}\left(C_{\alpha}\right)} \int_{C_{\alpha}} \theta_{0}\left(\sqrt{u_{1} / \pi}, \ldots, \sqrt{u_{d} / \pi}\right)^{s} d u_{1} \cdots d u_{d} .
\end{aligned}
$$

Since $\theta$ is continuous, the mean value theorem asserts that there is a point $z=$ $z(\alpha, s)=\left(z_{1}, z_{2}, \ldots, z_{d}\right) \in C_{\alpha}$ such that

$$
\tau_{\alpha, s}(\theta) \geq C_{0} \theta_{0}\left(\sqrt{z_{1} / \pi}, \ldots, \sqrt{z_{d} / \pi}\right)^{s} .
$$

Note that the point with coordinates $\zeta=\zeta(\alpha, s)=\left(\sqrt{z_{1} / \pi}, \ldots, \sqrt{z_{d} / \pi}\right)$ is in $D_{\alpha}$; consequently,

$$
\tau_{\alpha, s}(\theta) \geq C_{0} \theta_{0}(\zeta(\alpha, s))^{s} \quad \text { for } \zeta(\alpha, s) \in D_{\alpha}
$$

Finally

$$
\tau_{\alpha, s}(\theta) \tau_{\alpha, t}(\theta) \tau_{\alpha,-s-t}(\theta) \geq C_{0}^{3} \theta_{0}(\zeta)^{s} \theta_{0}\left(\zeta^{\prime}\right)^{t} \theta_{0}\left(\zeta^{\prime \prime}\right)^{-s-t}
$$

for points $\zeta, \zeta^{\prime}, \zeta^{\prime \prime} \in D_{\alpha}$. Since the weight $\theta$ is a $w$-moderate, $\theta_{0}$ satisfies

$$
\frac{\theta_{0}\left(z_{1}\right)}{\theta_{0}\left(z_{2}\right)} \geq \frac{1}{w\left(z_{1}-z_{2}\right)}, \quad z_{1}, z_{2} \in \mathbb{R}^{d} .
$$

Since $\zeta, \zeta^{\prime}, \zeta^{\prime \prime} \in D_{\alpha}$, the differences $\zeta-\zeta^{\prime \prime}$ and $\zeta^{\prime}-\zeta^{\prime \prime}$ are in the cube $\left[-\pi^{-1 / 2}, \pi^{-1 / 2}\right]^{d}$ as observed in (28). We conclude the non-trivial part of this estimate by

$$
\begin{aligned}
\tau_{\alpha, s}(\theta) \tau_{\alpha, t}(\theta) \tau_{\alpha,-s-t}(\theta) & \geq C_{0}^{3} \frac{1}{w\left(\zeta-\zeta^{\prime \prime}\right)^{s}} \frac{1}{w\left(\zeta^{\prime}-\zeta^{\prime \prime}\right)^{t}} \\
& \geq C_{0}^{3}\left(\max _{z \in\left[-\pi^{-1 / 2}, \pi^{-1 / 2}\right]^{d}} w(z)\right)^{-s-t}=C .
\end{aligned}
$$

The proof is complete.

The next result provides a sort of symbolic calculus for the anti-Wick operators $J_{\theta^{s}}$. Although the mapping $s \rightarrow J_{\theta^{s}}$ is not a homomorphism from $\mathbb{R}$ to operators, it is multiplicative modulo bounded operators.

Theorem 3.7. Let $\theta$ and $\mu$ be two moderate, continuous weight functions on $\mathbb{R}^{2 d}$ that are radial in each time-frequency variable. For every $r, s \in \mathbb{R}$ there exists an operator $V_{s, t}$ that is invertible on every $M_{\mu}^{2}\left(\mathbb{R}^{d}\right)$ such that

$$
J_{\theta^{s}} J_{\theta^{t}} J_{\theta^{-s-t}}=V_{s, t} \text {. }
$$


Proof. For $s, t \in \mathbb{R}$ fixed, set $\gamma(\alpha)=\tau_{\alpha, s}(\theta) \tau_{\alpha, t}(\theta) \tau_{\alpha,-s-t}(\theta)$ and

$$
V_{s, t} f=\sum_{\alpha \geq 0} \gamma(\alpha)\left\langle f, h_{\alpha}\right\rangle h_{\alpha}
$$

Clearly, $V_{s, t}=J_{\theta^{s}} J_{\theta^{t}} J_{\theta^{-s-t}}$. Since $C^{-1} \leq \gamma(\alpha) \leq C$ for all $\alpha \geq 0$ by Proposition 3.6. Proposition 3.3 implies that $V_{s, t}$ is bounded on every modulation space $M_{\mu}^{2}$. Likewise the formal inverse operator $V_{s, t}^{-1} f=\sum_{\alpha \geq 0} \gamma(\alpha)^{-1}\left\langle f, h_{\alpha}\right\rangle h_{\alpha}$ is bounded on $M_{\mu}^{2}\left(\mathbb{R}^{d}\right)$; consequently, $V_{s, t}$ is invertible on $M_{\mu}^{2}\left(\mathbb{R}^{d}\right)$.

We can now finish the proof of Theorem 3.2 .

Proof of Theorem 3.2. Choose $s=1$ and $t=-1$; then $J_{\theta} J_{1 / \theta}=V_{1,-1}$ is invertible on $L^{2}$. Similarly, the choice $s=-1, t=1$ yields that $J_{1 / \theta} J_{\theta}=V_{-1,1}$ is invertible on $M_{\theta}^{2}$. The factorization $J_{\theta} J_{1 / \theta}=V_{1,-1}$ implies that $J_{1 / \theta}$ is one-to-one from $L^{2}$ to $M_{\theta}^{2}$ and that $J_{\theta}$ maps $M_{\theta}^{2}$ onto $L^{2}$. The factorization $J_{1 / \theta} J_{\theta}=V_{-1,1}$ implies that $J_{\theta}$ is one-to-one from $M_{\theta}^{2}$ to $L^{2}$ and that $J_{1 / \theta}$ maps $L^{2}$ onto $M_{\theta}^{2}$.

We have proved that $J_{\theta}$ is an isomorphism from $M_{\theta}^{2}$ to $L^{2}$ and that $J_{1 / \theta}$ is an isomorphism from $L^{2}$ to $M_{\theta}^{2}$. The other isomorphisms are proved similarly.

Remark. In dimension $d=1$ the invertibility of $J_{\theta} J_{\theta^{-1}}$ follows from the equivalence $\tau_{n, 1}(\theta) \tau_{n,-1}(\theta) \asymp 1$, which can be expressed as the following inequality for weighted gamma functions:

$$
C^{-1} \leq \int_{0}^{\infty} \theta_{0}(\sqrt{x / \pi}) \frac{x^{n}}{n !} e^{-x} d x \int_{0}^{\infty} \frac{1}{\theta_{0}(\sqrt{x / \pi})} \frac{x^{n}}{n !} e^{-x} d x \leq C
$$

for all $n \geq 0$. Here $\theta_{0}$ is the same as before. It is a curious and fascinating fact that this inequality implies that the localization operator $J_{1 / \theta}$ is an isomorphism between $L^{2}(\mathbb{R})$ and $M_{\theta}^{2}(\mathbb{R})$.

\section{THE GENERAL ISOMORPHISM THEOREMS}

In Theorem 4.3 we will state the general isomorphism theorems. The strategy of the proof is similar to that of Theorem 3.2 in [21]. The main tools are the theorems about the spectral invariance of the generalized Sjöstrand classes [18, and the existence of a canonical isomorphism between $L^{2}\left(\mathbb{R}^{d}\right)$ and $M_{\theta}^{2}\left(\mathbb{R}^{d}\right)$ established in Theorem 3.2

4.1. Variations on spectral invariance. We first introduce the tools concerning the spectral invariance of pseudodifferential operators. Recall the following results from 18 .

Theorem 4.1. Let $v$ be a submultiplicative weight on $\mathbb{R}^{2 d}$ such that

$$
\lim _{n \rightarrow \infty} v(n z)^{1 / n}=1 \quad \text { for all } z \in \mathbb{R}^{2 d},
$$

and let $\tilde{v}$ be the same as in (10). If $T \in \mathrm{Op}\left(M_{\tilde{v}}^{\infty, 1}\right)$ and $T$ is invertible on $L^{2}\left(\mathbb{R}^{d}\right)$, then $T^{-1} \in \operatorname{Op}\left(M_{\tilde{v}}^{\infty, 1}\right)$.

Consequently, $T$ is invertible simultaneously on all modulation spaces $M_{\mu}^{p, q}\left(\mathbb{R}^{d}\right)$ for $1 \leq p, q \leq \infty$ and all $\mu \in \mathcal{M}_{v}$. 
Condition (31) is usually called the Gelfand-Raikov-Shilov (GRS) condition.

We prove a more general form of spectral invariance. Since we have formulated all results about the anti-Wick operators $J_{\theta}$ for radial weights only, we will assume from now on that all weights are radial in each coordinate. In this case

$$
\tilde{v}(x, \xi, \eta, y)=v(-\eta, y)=v(y, \eta),
$$

and we do not need the somewhat ugly distinction between $v$ and $\tilde{v}$.

Theorem 4.2. Assume that $v$ satisfies the GRS condition, $\theta^{2} \in \mathcal{M}_{v}$, and that both $v$ and $\theta$ are radial in each time-frequency coordinate.

If $T \in \mathrm{Op}\left(M_{v}^{\infty, 1}\right)$ and $T$ is invertible on $M_{\theta}^{2}\left(\mathbb{R}^{d}\right)$, then $T$ is invertible on $L^{2}\left(\mathbb{R}^{d}\right)$.

As a consequence $T$ is invertible on every modulation space $M_{\mu}^{p, q}\left(\mathbb{R}^{d}\right)$ for $1 \leq$ $p, q \leq \infty$ and $\mu \in \mathcal{M}_{v}$.

Proof. Set $\widetilde{T}=J_{\theta} T J_{1 / \theta}$. By Theorem 3.2, $J_{1 / \theta}$ is an isomorphism from $L^{2}\left(\mathbb{R}^{d}\right)$ onto $M_{\theta}^{2}\left(\mathbb{R}^{d}\right)$ and $J_{\theta}$ is an isomorphism from $M_{\theta}^{2}\left(\mathbb{R}^{d}\right)$ onto $L^{2}\left(\mathbb{R}^{d}\right)$; therefore $\widetilde{T}$ is an isomorphism on $L^{2}\left(\mathbb{R}^{d}\right)$.

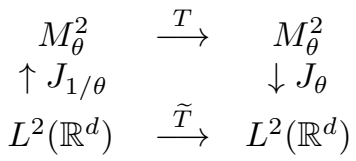

By Theorem 2.4 the operator $\widetilde{T}$ is in $\operatorname{Op}\left(M_{v^{1 / 2}}^{\infty, 1}\right)$. Since $\widetilde{T}$ is invertible on $L^{2}\left(\mathbb{R}^{d}\right)$, Theorem 4.1 on the spectral invariance of the symbol class $M_{v^{1 / 2}}^{\infty, 1}$ implies that the inverse operator $\widetilde{T}$ also possesses a symbol in $M_{v^{1 / 2}}^{\infty, 1}$, i.e., $\widetilde{T}^{-1} \in \operatorname{Op}\left(M_{v^{1 / 2}}^{\infty, 1}\right)$.

Now, since $\widetilde{T}^{-1}=J_{1 / \theta}^{-1} T^{-1} J_{\theta}^{-1}$, we find that

$$
T^{-1}=J_{1 / \theta} \widetilde{T}^{-1} J_{\theta} .
$$

Applying Theorem 2.4 once again, the symbol of $T^{-1}$ must be in $M^{\infty, 1}$. As a consequence, $T^{-1}$ is bounded on $L^{2}\left(\mathbb{R}^{d}\right)$.

Since $T \in \mathrm{Op}\left(M_{v}^{\infty, 1}\right)$ and $T$ is invertible on $L^{2}\left(\mathbb{R}^{d}\right)$, it follows that $T$ is also invertible on $M_{\mu}^{p, q}\left(\mathbb{R}^{d}\right)$ for every weight $\mu \in \mathcal{M}_{v}$ and $1 \leq p, q \leq \infty$.

4.2. An isomorphism theorem for localization operators. We now combine all steps and formulate and prove our main result, the isomorphism theorem for time-frequency localization operators with symbols of superfast growth.

Theorem 4.3. Let $g \in M_{v^{2} w}^{1}\left(\mathbb{R}^{d}\right), \mu \in \mathcal{M}_{w}$ and $m \in \mathcal{M}_{v}$ be such that $m$ is radial in each time-frequency coordinate and $v$ satisfies (31). Then the localization operator $A_{m}^{g}$ is an isomorphism from $M_{\mu}^{p, q}\left(\mathbb{R}^{d}\right)$ onto $M_{\mu / m}^{p, q}\left(\mathbb{R}^{d}\right)$ for $1 \leq p, q \leq \infty$.

Proof. Set $T=A_{1 / m}^{g} A_{m}^{g}$. We have already established that

(1) $T=A_{1 / m}^{g} A_{m}^{g}$ possesses a symbol in $M_{\tilde{v} \tilde{w}}^{\infty, 1}$ by Corollary 2.5 .

(2) $T$ is invertible on $M_{\theta}^{2}\left(\mathbb{R}^{d}\right)$ by Lemma 2.2

These are the assumptions of Theorem 4.2, and therefore $T$ is invertible on $M_{\mu}^{p, q}\left(\mathbb{R}^{d}\right)$ for every $\mu \in \mathcal{M}_{w} \subseteq \mathcal{M}_{v w}$ and $1 \leq p, q \leq \infty$. The factorization $T=A_{1 / m}^{g} A_{m}^{g}$ implies that $A_{m}^{g}$ is one-to-one from $M_{\mu}^{p, q}\left(\mathbb{R}^{d}\right)$ to $M_{\mu / m}^{p, q}\left(\mathbb{R}^{d}\right)$ and that $A_{1 / m}^{g}$ maps $M_{\mu / m}^{p, q}\left(\mathbb{R}^{d}\right)$ onto $M_{\mu}^{p, q}\left(\mathbb{R}^{d}\right)$. 
Now we change the order of the factors and consider the operator $T^{\prime}=A_{m}^{g} A_{1 / m}^{g}$ from $M_{\mu / m}^{p, q}\left(\mathbb{R}^{d}\right)$ to $M_{\mu / m}^{p, q}\left(\mathbb{R}^{d}\right)$ and factoring through $M_{\mu}^{p, q}\left(\mathbb{R}^{d}\right)$. Again $T^{\prime}$ possesses a symbol in $M_{\tilde{v} \tilde{w}}^{\infty, 1}$ and is invertible on $M_{1 / \theta}^{2}\left(\mathbb{R}^{d}\right)$. With Theorem 4.2 we conclude that $T^{\prime}$ is invertible on all modulation spaces $M_{\mu / m}^{p, q}\left(\mathbb{R}^{d}\right)$. The factorization of $T^{\prime}=A_{m}^{g} A_{1 / m}^{g}$ now yields that $A_{1 / m}^{g}$ is one-to-one from $M_{\mu / m}^{p, q}\left(\mathbb{R}^{d}\right)$ to $M_{\mu}^{p, q}\left(\mathbb{R}^{d}\right)$ and that $A_{m}^{g}$ maps $M_{\mu}^{p, q}\left(\mathbb{R}^{d}\right)$ onto $M_{\mu / m}^{p, q}\left(\mathbb{R}^{d}\right)$.

As a consequence $A_{m}^{g}$ is bijective from $M_{\mu}^{p, q}\left(\mathbb{R}^{d}\right)$ onto $M_{\mu / m}^{p, q}\left(\mathbb{R}^{d}\right)$ and $A_{1 / m}^{g}$ is bijective from $M_{\mu / m}^{p, q}\left(\mathbb{R}^{d}\right)$ onto $M_{\mu}^{p, q}\left(\mathbb{R}^{d}\right)$.

\section{Consequences for Gabor multipliers and Toeplitz operators ON THE BARGMANN-FOCK SPACE}

5.1. Gabor multipliers. Gabor multipliers are time-frequency localization operators whose symbols are discrete measures. Their basic properties are the same as for the corresponding localization operators, but the discrete definition makes them more accessible for numerical computations.

Let $\Lambda=A \mathbb{Z}^{2 d}$ for some $A \in \mathrm{GL}(2 d, \mathbb{R})$ be a lattice in $\mathbb{R}^{2 d}$, let $g, \gamma$ be suitable window functions (as in Proposition 5.1) and $m$ be a weight sequence defined on $\Lambda$. Then the Gabor multiplier $G_{m}^{g, \gamma, \Lambda}$ is defined to be

$$
G_{m}^{g, \gamma, \Lambda} f=\sum_{\lambda \in \Lambda} m(\lambda)\langle f, \pi(\lambda) g\rangle \pi(\lambda) \gamma
$$

The boundedness of Gabor multipliers between modulation spaces is formulated and proved exactly as for time-frequency localization operators. See [15] for a detailed exposition of Gabor multipliers and [10] for general boundedness results that include distributional symbols.

Proposition 5.1. Let $g, \gamma \in M_{v w}^{1}\left(\mathbb{R}^{d}\right)$, and let $m$ be a continuous moderate weight function $m \in \mathcal{M}_{v}$. Then $G_{m}^{g, \gamma, \Lambda}$ is bounded from $M_{\mu}^{p, q}\left(\mathbb{R}^{2 d}\right)$ to $M_{\mu / m}^{p, q}\left(\mathbb{R}^{d}\right)$.

In the following we will assume that the set $\mathcal{G}(g, \Lambda)=\{\pi(\lambda) g: \lambda \in \Lambda\}$ is a Gabor frame for $L^{2}\left(\mathbb{R}^{d}\right)$. This means that the frame operator $S_{g, \Lambda} f=M_{1}^{g, g, \Lambda}=$ $\sum_{\lambda \in \Lambda}\langle f, \pi(\lambda) g\rangle \pi(\lambda) g$ is invertible on $L^{2}\left(\mathbb{R}^{d}\right)$. From the rich theory of Gabor frames we quote only the following result: If $g \in M_{v}^{1}$ and $\mathcal{G}(g, \Lambda)$ is a frame for $L^{2}\left(\mathbb{R}^{d}\right)$, $1 \leq p \leq \infty$, and $m \in \mathcal{M}_{v}$, then

$$
\|f\|_{M_{m}^{p}} \asymp\left(\sum_{\lambda \in \Lambda}|\langle f, \pi(\lambda) g\rangle|^{p} m(\lambda)^{p}\right)^{1 / p} \quad \text { for all } f \in M_{m}^{p}\left(\mathbb{R}^{d}\right) .
$$

We refer to [17] for a detailed discussion, the proof, and for further references.

Our main result on Gabor multipliers is the isomorphism theorem.

Theorem 5.2. Assume that $g \in M_{v^{2} w}^{1}\left(\mathbb{R}^{d}\right)$ and that $\mathcal{G}(g, \Lambda)$ is a frame for $L^{2}\left(\mathbb{R}^{d}\right)$. If $m \in \mathcal{M}_{v}$ is radial in each time-frequency coordinate, then $G_{m}^{g, g, \Lambda}$ is an isomorphism from $M_{\mu}^{p, q}\left(\mathbb{R}^{2 d}\right)$ onto $M_{\mu / m}^{p, q}\left(\mathbb{R}^{d}\right)$ for every $\mu \in \mathcal{M}_{w}$ and $1 \leq p, q \leq \infty$.

The proof is similar to the proof of Theorem 4.3, and we only sketch the necessary modifications. In the following we fix the lattice $\Lambda$ and choose $\gamma=g$ and drop the reference to these additional parameters by writing $G_{m}^{g, \gamma, \Lambda}$ as $G_{m}^{g}$ in analogy to the localization operator $A_{m}^{g}$. 
Proposition 5.3. If $g \in M_{v}^{1}\left(\mathbb{R}^{d}\right)$ and $m \in \mathcal{M}_{v}$ and $\theta=m^{1 / 2}$, then $G_{m}^{g}$ is an isomorphism from $M_{\theta}^{2}\left(\mathbb{R}^{d}\right)$ onto $M_{1 / \theta}^{2}\left(\mathbb{R}^{d}\right)$.

Proof. By Proposition $5.1 G_{m}^{g}$ is bounded from $M_{\theta}^{2}$ to $M_{\theta / m}^{2}=M_{1 / \theta}^{2}$, and thus

$$
\left\|G_{m}^{g} f\right\|_{M_{1 / \theta}^{2}} \leq C\|f\|_{M_{\theta}^{2}} .
$$

Next we use the characterization of $M_{\theta}^{2}$ by Gabor frames and relate it to Gabor multipliers:

$$
\left\langle G_{m}^{g} f, f\right\rangle=\sum_{\lambda \in \Lambda} m(\lambda)|\langle f, \pi(\lambda) g\rangle|^{2} \asymp\|f\|_{M_{\theta}^{2}}^{2} .
$$

This identity implies that $G_{m}^{g}$ is one-to-one on $M_{\theta}^{2}$ and that $\left\|G_{m}^{g} f\right\|_{M_{1 / \theta} \text { is an }}$ equivalent norm on $M_{\theta}^{2}$. Since $G_{m}^{g}$ is self-adjoint, it has dense range in $M_{1 / \theta}^{2}$, whence $G_{m}^{g}$ is onto as well.

Using the weight $1 / m$ instead of $m$ and $1 / \theta$ instead of $\theta$, we see that $G_{1 / m}^{g}$ is an isomorphism from $M_{1 / \theta}^{2}\left(\mathbb{R}^{d}\right)$ onto $M_{\theta}^{2}\left(\mathbb{R}^{d}\right)$.

Proof of Theorem 5.2. We proceed as in the proof of Theorem 4.3. Define the operators $T=G_{1 / m}^{g} G_{m}^{g}$ and $T^{\prime}=G_{m}^{g} G_{1 / m}^{g}$. By Proposition $5.1 T$ maps $M_{\theta}^{2}\left(\mathbb{R}^{d}\right)$ to $M_{\theta}^{2}\left(\mathbb{R}^{d}\right)$, and since $T$ is a composition of two isomorphisms, $T$ is an isomorphism on $M_{\theta}^{2}\left(\mathbb{R}^{d}\right)$. Likewise $T^{\prime}$ is an isomorphism on $M_{1 / \theta}^{2}\left(\mathbb{R}^{d}\right)$.

As in Corollary 2.5 we verify that the symbol of $T$ is in $M_{\tilde{v} \tilde{w}}^{\infty, 1}\left(\mathbb{R}^{2 d}\right)$. Theorem 4.2 then asserts that $T$ is invertible on $L^{2}\left(\mathbb{R}^{d}\right)$, and the general spectral invariance implies that $T$ is an isomorphism on $M_{\mu}^{p, q}\left(\mathbb{R}^{d}\right)$. Likewise $T^{\prime}$ is an isomorphism on $M_{\mu / m}^{p, q}\left(\mathbb{R}^{d}\right)$. This means that each of the factors of $T$ must be an isomorphism on the correct space.

5.2. Toeplitz operators. The Bargmann-Fock space $\mathcal{F}=\mathcal{F}^{2}\left(\mathbb{C}^{d}\right)$ is the Hilbert space of all entire functions of $d$ variables such that

$$
\|F\|_{\mathcal{F}}^{2}=\int_{\mathbb{C}^{d}}|F(z)|^{2} e^{-\pi|z|^{2}} d z<\infty
$$

Here $d z$ is the Lebesgue measure on $\mathbb{C}^{d}=\mathbb{R}^{2 d}$.

Related to the Bargmann-Fock space $\mathcal{F}^{2}$ are spaces of entire functions satisfying weighted integrability conditions. Let $m$ be a moderate weight on $\mathbb{C}^{d}$ and $1 \leq$ $p, q \leq \infty$. Then the space $\mathcal{F}_{m}^{p, q}\left(\mathbb{C}^{d}\right)$ is the Banach space of all entire functions of $d$ complex variables such that

$$
\|F\|_{\mathcal{F}_{m}^{p, q}}^{q}=\int_{\mathbb{R}^{d}}\left(\int_{\mathbb{R}^{d}}|F(x+i y)|^{p} m(x+i y)^{p} e^{-p \pi|x+i y|^{2} / 2} d x\right)^{q / p} d y<\infty .
$$

Let $P$ be the orthogonal projection from $L^{2}\left(\mathbb{C}^{d}, \mu\right)$ with Gaussian measure $d \mu(z)=e^{-\pi|z|^{2}} d z$ onto $\mathcal{F}^{2}\left(\mathbb{C}^{d}\right)$. Then $P$ is given by the formula

$$
P F(w)=\int_{\mathbb{C}^{d}} F(z) e^{\pi \bar{z} w} e^{-\pi|z|^{2}} d z .
$$

We remark that a function on $\mathbb{R}^{2 d}$ with a certain growth is entire if and only if it satisfies $F=P F$. 
The classical Toeplitz operator on Bargmann-Fock space with a symbol $m$ is defined by $T_{m} F=P(m F)$ for $F \in \mathcal{F}^{2}\left(\mathbb{C}^{d}\right)$. Explicitly $T_{m}$ is given by the formula

$$
T_{m} F(w)=\int_{\mathbb{C}^{d}} m(z) F(z) e^{\pi \bar{z} w} e^{-\pi|z|^{2}} d z .
$$

See [2, 3, 4, 15, 12, 16] for a sample of references.

In the following we assume that the symbol of a Toeplitz operator is continuous and radial in each coordinate, i.e., $m\left(z_{1}, \ldots, z_{d}\right)=m_{0}\left(\left|z_{1}\right|, \ldots,\left|z_{d}\right|\right)$ for some continuous function $m_{0}$ from $\mathbb{R}_{+}^{d}$ to $\mathbb{R}_{+}$.

Theorem 5.4. Let $\mu \in \mathcal{M}_{w}$, and let $m \in \mathcal{M}_{v}$ be a continuous moderate weight function such that one of the following conditions is fulfilled:

(i) Either $m$ is radial in each coordinate

(ii) or $m$ is of polynomial type.

Then the Toeplitz operator $T_{m}$ is an isomorphism from $\mathcal{F}_{\mu}^{p, q}\left(\mathbb{C}^{d}\right)$ onto $\mathcal{F}_{\mu / m}^{p, q}\left(\mathbb{C}^{d}\right)$ for $1 \leq p, q \leq \infty$.

The formulation of Theorem 5.4 looks similar to the main theorem about timefrequency localization operators. In fact, after a suitable translation of concepts, it is a special case of Theorem 4.3 .

To explain the connection, we recall the Bargmann transform that maps distributions on $\mathbb{R}^{d}$ to entire functions on $\mathbb{C}^{d}$ :

$$
\mathcal{B} f(z)=F(z)=2^{d / 4} e^{-\pi z^{2} / 2} \int_{\mathbb{R}^{d}} f(t) e^{-\pi t^{2}} e^{2 \pi t \cdot z} d t, \quad z \in \mathbb{C}^{d} .
$$

If $f$ is a distribution, then we interpret the integral as the action of $f$ on the function $e^{-\pi t^{2}} e^{2 \pi t \cdot z}$.

The connection to time-frequency analysis comes from the fact that the Bargmann transform is just a short-time Fourier transform in disguise [17, Ch. 3]. As before, we use the normalized Gaussian $h(t)=2^{d / 4} e^{-\pi t^{2}}$ as a window. Identifying the pair $(x, \xi) \in \mathbb{R}^{d} \times \mathbb{R}^{d}$ with the complex vector $z=x+i \xi \in \mathbb{C}^{d}$, the short-time Fourier transform of a function $f$ on $\mathbb{R}^{d}$ with respect to $h$ is

$$
V_{h} f(\bar{z})=e^{\pi i x \cdot \xi} \mathcal{B} f(z) e^{-\pi|z|^{2} / 2} .
$$

In particular, the Bargmann transform of the time-frequency shift $\pi(w) h$ is given by

$$
\mathcal{B}(\pi(w) h)(z)=e^{-\pi i u \cdot \eta} e^{\pi \bar{w} z} e^{-\pi|w|^{2} / 2}, \quad w, z \in \mathbb{C}^{d}, w=u+i \eta .
$$

It is a basic fact that the Bargmann transform is a unitary mapping from $L^{2}(\mathbb{R})$ onto the Bargmann-Fock space $\mathcal{F}^{2}\left(\mathbb{C}^{d}\right)$. Furthermore, the Hermite functions $h_{\alpha}, \alpha \geq 0$, are mapped to the normalized monomials $e_{\alpha}(z)=\pi^{|\alpha| / 2}(\alpha !)^{-1 / 2} z^{\alpha}$.

Let $m^{\prime}(z)=m(\bar{z})$. Then (41) implies that the Bargmann transform $\mathcal{B}$ maps $M_{m}^{p, q}\left(\mathbb{R}^{d}\right)$ isometrically to $\mathcal{F}_{m^{\prime}}^{p, q}\left(\mathbb{C}^{d}\right)$. By straightforward arguments it follows that the Bargmann transform maps the modulation space $M_{m}^{p, q}\left(\mathbb{R}^{d}\right)$ onto the Fock space $\mathcal{F}_{m^{\prime}}^{p, q}\left(\mathbb{C}^{d}\right)[17,28$.

The connection between time-frequency localization operators and Toeplitz operators on the Bargmann-Fock space is given by the following statement. 
Proposition 5.5. Let $m$ be a moderate weight function on $\mathbb{R}^{2 d} \simeq \mathbb{C}^{d}$ and set $m^{\prime}(z)=m(\bar{z})$. The Bargmann transform intertwines $J_{m}$ and $T_{m^{\prime}}$, i.e., for all $f \in L^{2}\left(\mathbb{R}^{d}\right)$ (or $\left.f \in\left(S_{1 / 2}^{1 / 2}\right)^{*}\right)$ we have

$$
\mathcal{B}\left(J_{m} f\right)=T_{m^{\prime}}(\mathcal{B} f) .
$$

Proof. This fact is well known [8, 12. For completeness and consistency of notation, we provide the formal calculation.

We take the short-time Fourier transform of $J_{m}=A_{m}^{h}$ with respect to $h$. On the one hand we obtain that

$$
\left\langle J_{m} f, \pi(\bar{w}) h\right\rangle=e^{\pi i u \cdot \eta} \mathcal{B}\left(J_{m} f\right)(w) e^{-\pi|w|^{2} / 2},
$$

and on the other hand, after substituting (41) and (42), we obtain

$$
\begin{aligned}
\left\langle J_{m} f, \pi(\bar{w}) h\right\rangle & =\int_{\mathbb{R}^{2 d}} m(\bar{z}) V_{h} f(\bar{z}) \overline{V_{h}(\pi(\bar{w}) h)(\bar{z})} d z \\
& =\int_{\mathbb{C}^{d}} m(\bar{z}) \mathcal{B} f(z) \overline{\mathcal{B}(\pi(\bar{w}) h)(\bar{z})} e^{-\pi|z|^{2}} d z \\
& =e^{\pi i u \cdot \eta} \int_{\mathbb{C}^{d}} m(\bar{z}) \mathcal{B} f(z) e^{\pi \bar{z} w} e^{-\pi|z|^{2}} d z e^{-\pi|w|^{2} / 2} .
\end{aligned}
$$

Comparing (44) and (45) we obtain

$$
\mathcal{B}\left(J_{m} f\right)(w)=\int_{\mathbb{C}^{d}} m(\bar{z}) \mathcal{B} f(z) e^{\pi \bar{z} w} e^{-\pi|z|^{2}} d z=T_{m^{\prime}} \mathcal{B} f(w) .
$$

Proof of Theorem 5.4. First assume that (i) holds, i.e., $m$ is radial in each variable. The Bargmann transform is an isomorphism between the modulation space $M_{m}^{p, q}\left(\mathbb{R}^{d}\right)$ and the Bargmann-Fock space $\mathcal{F}_{m^{\prime}}^{p, q}\left(\mathbb{C}^{d}\right)$ for arbitrary moderate weight function $m$ and $1 \leq p, q \leq \infty$. By Theorem 4.3 the anti-Wick operator $J_{m}$ is an isomorphism from $M_{\mu}^{p, q}\left(\mathbb{R}^{d}\right)$ onto $M_{\mu / m}^{p, q}\left(\mathbb{R}^{d}\right)$. Since $T_{m^{\prime}}$ is a composition of three isomorphisms (Proposition [5.5 and (46)), the Toeplitz operator $T_{m^{\prime}}$ is an isomorphism from $\mathcal{F}_{\mu^{\prime}}^{p, q}\left(\mathbb{C}^{d}\right)$ onto $\mathcal{F}_{\mu^{\prime} / m^{\prime}}^{p, q}\left(\mathbb{C}^{d}\right)$ :

$$
\begin{array}{ccc}
\mathcal{F}_{\mu^{\prime}}^{p, q} & \stackrel{T_{m^{\prime}}}{\longrightarrow} & \mathcal{F}_{\mu^{\prime} / m^{\prime}}^{p, q} \\
\uparrow \mathcal{B} & & \uparrow \mathcal{B} \\
M_{\mu}^{p, q} & \stackrel{J_{m}}{\longrightarrow} & M_{\mu / m}^{p, q}
\end{array}
$$

Finally replace $m^{\prime}$ and $\mu^{\prime}$ by $m$ and $\mu$.

By using Theorem 3.2 in 21 instead of Theorem 4.3, the same arguments show that the result follows when (ii) is fulfilled.

\section{REFERENCES}

[1] H. Ando and Y. Morimoto. Wick calculus and the Cauthy problem for some dispersive equations. Osaka J. Math., 39(1):123-147, 2002. MR.1883917 (2003b:35219)

[2] V. Bargmann. On a Hilbert space of analytic functions and an associated integral transform. Comm. Pure Appl. Math., 14:187-214, 1961. MR0157250 (28:486)

[3] F. A. Berezin. Wick and anti-Wick symbols of operators. Mat. Sb. (N.S.), 86(128):578-610, 1971. MR0291839(45:929)

[4] C. A. Berger and L. A. Coburn. Toeplitz operators on the Segal-Bargmann space. Trans. Amer. Math. Soc., 301(2):813-829, 1987. MR882716 (88c:47044)

[5] C. A. Berger and L. A. Coburn. Heat flow and Berezin-Toeplitz estimates. Amer. J. Math., 116(3):563-590, 1994. MR1277446 (95g:47038) 
[6] P. Boggiatto, E. Cordero, and K. Gröchenig. Generalized anti-Wick operators with symbols in distributional Sobolev spaces. Integral Equations Operator Theory, 48(4):427-442, 2004. MR2047590(2005a:47088)

[7] J.-M. Bony and J.-Y. Chemin. Espaces fonctionnels associés au calcul de Weyl-Hörmander. Bull. Soc. Math. France, 122(1):77-118, 1994. MR.1259109 (95a:35152)

[8] L. A. Coburn. The Bargmann isometry and Gabor-Daubechies wavelet localization operators. In Systems, approximation, singular integral operators, and related topics (Bordeaux, 2000), volume 129 of Oper. Theory Adv. Appl., pages 169-178. Birkhäuser, Basel, 2001. MR.1882695 (2003a:47054)

[9] J. B. Conway. A Course in Functional Analysis. Springer-Verlag, New York, second edition, 1990. MR1070713 (91e:46001)

[10] E. Cordero and K. Gröchenig. Time-frequency analysis of localization operators. J. Funct. Anal., 205(1):107-131, 2003. MR2020210 (2004j:47100)

[11] E. Cordero and K. Gröchenig. Symbolic calculus and Fredholm property for localization operators. J. Fourier Anal. Appl., 12(4):345-370, 2006. MR2256930 (2007e:47030)

[12] I. Daubechies. Time-frequency localization operators: A geometric phase space approach. IEEE Trans. Inform. Theory, 34(4):605-612, 1988. MR966733

[13] M. Engliš. Toeplitz operators and localization operators. Trans. Amer. Math. Soc., 361(2):1039-1052, 2009. MR2452833 (2010a:47056)

[14] H. G. Feichtinger and K. Gröchenig. Banach spaces related to integrable group representations and their atomic decompositions. I. J. Funct. Anal., 86(2):307-340, 1989. MR.1021139 (91g:43011)

[15] H. G. Feichtinger and K. Nowak. A first survey of Gabor multipliers. In Advances in Gabor analysis, Appl. Numer. Harmon. Anal., pages 99-128. Birkhäuser Boston, Boston, MA, 2003. MR.1955933

[16] G. B. Folland. Harmonic Analysis in Phase Space. Princeton Univ. Press, Princeton, NJ, 1989. MR983366 (92k:22017)

[17] K. Gröchenig. Foundations of Time-Frequency Analysis. Birkhäuser Boston, Inc., Boston, MA, 2001. MR:1843717 (2002h:42001)

[18] K. Gröchenig. Time-frequency analysis of Sjöstrand's class. Revista Mat. Iberoam., 22(2):703724, 2006. MR2294795 (2008b:35308)

[19] K. Gröchenig. Weight functions in time-frequency analysis. Pseudodifferential Operators: Partial Differential Equations and Time-Frequency Analysis, (English Summary), volume 52, pages 343-366. Fields Institute Comm., 2007. MR2385335 (2009c:42070)

[20] K. Gröchenig and Z. Rzeszotnik. Banach algebras of pseudodifferential operators and their almost diagonalization. Ann. Inst. Fourier (Grenoble), 58(7):2279-2314, 2008. MR2498351 (2010h:47071)

[21] K. Gröchenig and J. Toft. Isomorphism properties of Toeplitz operators and pseudodifferential operators between modulation spaces. J. Anal. Math., 114 (1):255-283, 2011. MR.2837086

[22] A. Holst, J. Toft and P. Wahlberg. Weyl product algebras and modulation spaces. J. Funct. Anal., 251:463-491, 2007. MR2356420(2008i:42047)

[23] A. J. E. M. Janssen. Bargmann transform, Zak transform, and coherent states. J. Math. Phys., 23(5):720-731, 1982. MR655886 (84h:81041)

[24] N. Lerner. The Wick calculus of pseudo-differential operators and energy estimates. In New trends in microlocal analysis (Tokyo, 1995), pages 23-37. Springer, Tokyo, 1997. MR1636234 (99i:35187)

[25] N. Lerner. The Wick calculus of pseudo-differential operators and some of its applications. Cubo Mat. Educ., 5(1):213-236, 2003. MR1957713 (2004a:47058)

[26] N. Lerner and Y. Morimoto. A Wiener algebra for the Fefferman-Phong inequality. In Seminaire: Equations aux Dérivées Partielles. 2005-2006, Sémin. Équ. Dériv. Partielles, Exp. No. XVII, 12pp. École Polytech., Palaiseau, 2006. MR2276082 (2008c:47077)

[27] M. A. Shubin. Pseudodifferential Operators and Spectral Theory. Springer-Verlag, Berlin, second edition, 2001. Translated from the 1978 Russian original by Stig I. Andersson. MR:1852334 (2002d:47073)

[28] M. Signahl and J. Toft. Remarks on mapping properties for the Bargmann transform on modulation spaces. Integral Transforms Spec. Funct., 22(4-5):359-366, 2011. MR2801288 (2012e:44006) 
[29] S. Thangavelu. Lectures on Hermite and Laguerre Expansions, volume 42 of Mathematical Notes. Princeton University Press, Princeton, NJ, 1993. With a preface by Robert S. Strichartz. MR1215939 (94i:42001)

[30] J. Toft. Regularizations, decompositions and lower bound problems in the Weyl calculus. Comm. Partial Differential Equations, 25 (7\&8): 1201-1234, 2000. MR1765143(2001i:47081)

[31] J. Toft. Subalgebras to a Wiener type algebra of pseudo-differential operators. Ann. Inst. Fourier (Grenoble), 51(5):1347-1383, 2001. MR1860668(2002h:47071)

[32] J. Toft. Continuity properties for modulation spaces, with applications to pseudo-differential calculus. I. J. Funct. Anal., 207(2):399-429, 2004. MR2032995(2004j:35312)

[33] J. Toft. Continuity and Schatten-von Neumann properties for Toeplitz operators on modulation spaces. In: J. Toft, M. W. Wong, H. Zhu (Eds.), Modern Trends in Pseudo-Differential Operators, Operator Theory Advances and Applications, Vol. 172, Birkhäuser Verlag, Basel: pp. 313-328, 2007. MR2308518 (2008d:47064)

[34] J. Toft. Multiplication properties in pseudo-differential calculus with small regularity on the symbols. J. Pseudo-Differ. Oper. Appl. 1(1): 101-138, 2010. MR2679745 (2011h:47094)

[35] H. Triebel. Theory of Function Spaces. Birkhäuser Verlag, Basel, 1983. MR781540(86j:46026)

[36] M. W. Wong. Wavelet Transforms and Localization Operators, volume 136 of Operator Theory Advances and Applications. Birkhäuser, 2002. MR1918652 (2003i:42003)

Faculty of Mathematics, University of Vienna, Nordbergstrasse 15, A-1090 Vienna, Austria

E-mail address: karlheinz.groechenig@univie.ac.at

Department of Computer Science, Physics and Mathematics, Linnæus University, VÄxJÖ, SWEDEN

E-mail address: joachim.toft@lnu.se 